\title{
Dimensões gerenciais na formação acadêmica de enfermagem: uma revisão integrativa
}

\author{
Raissa Mont'Alverne Barreto ${ }^{1}$, \\ Mayara Nascimento Vasconcelos ${ }^{2}$, \\ Etelvina Sampaio Melo $^{3}$, \\ Maria Auxiliadora Ferreira Araújo ${ }^{4}$, \\ Roberta Cavalcante Muniz Lira ${ }^{5}$, \\ Izabelle Mont'Alvene Napoleão Albuquerque ${ }^{6}$
}

\section{RESUMO}

Objetivou-se realizar um levantamento da produção científica sobre o processo de formação do enfermeiro para o desenvolvimento de habilidades gerenciais na Atenção Primária à Saúde. Trata-se de uma revisão integrativa nas bases de dados LILACS, BDENF, MEDLINE e PUBMED, resultando em 14 artigos para análise. Evidenciaram-se lacunas existentes na formação do enfermeiro para habilidades gerenciais. Entretanto, a literatura aponta que, apesar desses desafios, o enfermeiro continua sendo o profissional de saúde mais capacitado para exercer cargos de gestão. As produções refletem a necessidade de educação permanente aos profissionais para reorientação das práticas de trabalho a fim de contemplar suas atividades gerenciais. Assim, enfatiza-se a importância da dimensão gerencial de forma mais significativa no processo de formação que leve ao alcance de metas e à construção de uma prática integral, autônoma e resolutiva para o exercício de uma gerência competente frente às suas ações e responsabilidades.

Descritores: Estratégia Saúde da Família; Educação em Enfermagem; Gestão em Saúde.

\footnotetext{
${ }^{1}$ Enfermeira. Discente do Programa de Pós-Graduação em Saúde da Família da Universidade Federal do Ceará, nível Mestrado. Sobral, CE, Brasil. Email: raissinha_@hotmail.com.

2 Enfermeira. Discente do Programa de Pós-Graduação em Saúde da Família da Universidade Federal do Ceará, nível Mestrado. Sobral, CE, Brasil. Email: mayaravasconcelos92@hotmail.com.

${ }^{3}$ Enfermeira. Discente do Programa de Pós-Graduação em Saúde da Família da Universidade Federal do Ceará, nível Mestrado. Sobral, CE, Brasil. Email: etelvinamelo@hotmail.com.

${ }^{4}$ Psicóloga. Discente do Programa de Pós-Graduação em Saúde da Família da Universidade Federal do Ceará, nível Mestrado. Sobral, CE, Brasil. Email: sulypsico@yahoo.com.br.

${ }^{5}$ Enfermeira, Doutora em Enfermagem. Professora Adjunto da Universidade Federal do Ceará. Sobral, CE, Brasil. E-mail: rcavalcantemunizlira0@gmail.com.

${ }^{6}$ Enfermeira, Doutora em Enfermagem. Professora Adjunto da Universidade Estadual Vale do Acaraú. Sobral, CE, Brasil. E-mail: izabellemontalverne@gmail.com.
}

Artigo recebido: $21 / 07 / 2017$.

Artigo aprovado: 26/06/2018.

Artigo publicado: 29/11/2018.

\section{Como citar esse artigo:}

Barreto RMA, Vasconcelos MN, Melo ES, Araújo MAF, Lira RCM, Albuquerque IMAN. Dimensões gerenciais na formação acadêmica de enfermagem: uma revisão integrativa. Rev. Eletr. Enf. [Internet]. 2018 [acesso em:

];20:v20a27. Disponível em: https://doi.org/10.5216/ree.v20.47945. 


\section{INTRODUÇÃO}

O Programa de Saúde da Família (PSF) corresponde a uma política pública de saúde que teve início em 1994 sob a iniciativa do Ministério da Saúde (MS). Apesar do termo "programa", a nomenclatura Estratégia Saúde da Família (ESF) foi assumida por se configurar como estratégia estruturante de reorientação da Atenção Primária à Saúde (APS) do Sistema Único de Saúde (SUS) ${ }^{(1)}$.

Assim, os desafios para a qualificação do SUS e os modelos de atenção que dele derivam são muitos e variados, passando pela formação e capacitação dos profissionais que integram a rede pública de assistência à saúde ${ }^{(2)}$.

Apesar de alguns avanços, estudiosos revelam que o processo formativo dos profissionais de saúde ainda está muito distante do cuidado integral e, além disso, o perfil destes trabalhadores demonstra qualificação insuficiente para as mudanças das práticas ${ }^{(3)}$.

Diante desta fragilidade, a formação de enfermeiros para o campo de trabalho é um importante aspecto a ser discutido, a qual é entendida aqui como um processo que visa a construção de competências que deve ocorrer em cenários de desafios complexos, considerando a globalização, o incremento da competitividade profissional e os problemas de saúde que são exacerbados pelas constantes mudanças no contexto político e econômico do País ${ }^{(4-5)}$.

Nessa perspectiva, as instituições de ensino têm buscado formas de abranger em seus currículos os conteúdos e as práticas necessários para a formação dos profissionais que irão atuar no SUS, entretanto, as mudanças promovidas no ensino não correspondem suficientemente às exigências do mercado de trabalho(6).

Além disso, as demandas do SUS requerem da educação dos profissionais de saúde a adequação de habilidades relacionadas à gestão e gerência, uma vez que as Diretrizes Curriculares Nacionais, para a graduação em Enfermagem, orientam a formação de profissionais generalistas, humanistas, críticos e reflexivos, capazes de avaliar, sistematizar, decidir as condutas mais adequadas baseadas em evidências científicas e estar aptos a serem empreendedores, gestores, empregadores ou lideranças na equipe de saúde ${ }^{(7)}$.

Estudiosos revelam que a APS é marcada por problemas de gestão que se refletem na sua estrutura física, nos serviços, perfil dos gestores e trabalhadores, no acesso aos recursos e efetividade das políticas. Prestar serviços com excelência se constitui essencial na qualidade da gestão. Então, a gerência da Unidade Básica de Saúde (UBS) é um instrumento necessário e importante no cuidado em saúde, uma vez que produz certa direcionalidade ao processo de trabalho( ${ }^{(8)}$, e o enfermeiro assume participação significativa neste cargo, dada sua atuação no compromisso com a saúde dos indivíduos e da coletividade, e o fato de se relacionarem de maneira satisfatória com os demais membros da equipe multiprofissional(9).

A literatura científica revela ainda que o enfermeiro, enquanto gerente do cuidado, mobiliza ações nas relações e interações entre as pessoas como indivíduos complexos e que vivenciam a organicidade do sistema de cuidado com aptidões gerenciais próprias ou inerentes às atividades profissionais dos enfermeiros, por meio do planejamento das ações de cuidado, da previsão e provisão de recursos necessários para organização da assistência de uma UBS ${ }^{(10)}$.

Porém, considerando a premissa do contexto atual do trabalho em saúde, autores reconhecem que o desenvolvimento de habilidades do enfermeiro que possam sustentar uma prática gerencial qualificada é um 
desafio, principalmente pela formação que, na maioria das instituições, ainda tem o foco voltado para o desenvolvimento de ações técnico-assistencialistas ${ }^{(3,11)}$.

Assim, o descompasso entre o processo de formação e a prática do enfermeiro resulta em tensões, desmotivação e conflitos ${ }^{(10)}$. A escolha por conteúdos no gerenciamento em Enfermagem relaciona-se às funções diferenciadas que os enfermeiros vêm desenvolvendo no cenário da saúde no País, de forma a assumir progressivamente cargos de gestão(12), justificando, assim, o fato de os estudos desta pesquisa não se limitarem apenas à gestão da UBS, ou gestão do cuidado, ou gestão de processos de trabalho, mas de englobarem pesquisas e discussões sobre gestão no sentido mais amplo no âmbito da APS.

Nessa perspectiva, pesquisadores desta temática apontam para a necessidade de reflexões e discussões constantes sobre as diferentes formas de gestão ${ }^{(5,13)}$ que o enfermeiro vem desempenhando na APS, considerando que esta é a porta de entrada do SUS, onde uma administração de qualidade dos processos de trabalho, do cuidado e da gerência da UBS pode definir mudanças no modo de fazer saúde ${ }^{(14)}$.

Além disso, considera-se relevante o reconhecimento e caracterização da formação do enfermeiro para habilidades de gestão na APS, partindo do pressuposto que a contribuição pretendida, a partir desse estudo, segue no sentido de sustentar discussões que busquem fornecer subsídios para remodelação dos modelos de formação que vá ao encontro das necessidades de reordenação no modelo de gestão e atenção.

Dessa forma, delineou-se a seguinte questão norteadora: Quais as evidências na literatura científica que direcionam a formação do enfermeiro para habilidades gerenciais no âmbito da Estratégia Saúde da Família?

Em meio a toda esta efervescência dos processos formativos e de atenção à saúde, este estudo buscou realizar um levantamento da produção científica sobre o processo de formação do enfermeiro para o desenvolvimento de habilidades gerenciais na Atenção Primária à Saúde.

\section{METODOLOGIA}

Trata-se de uma revisão integrativa, a qual permite incluir estudos com diferentes abordagens metodológicas, agrupando resultados obtidos de um conjunto de pesquisas primárias com temáticas idênticas ou similares $^{(15)}$.

A busca foi realizada por meio da Biblioteca Virtual de Saúde (BIREME), no período de maio e junho de 2017, que incluíram as seguintes bases de dados: Literatura Internacional em Ciências da Saúde (MEDLINE), Literatura Latino Americana e do Caribe em Ciências da Saúde (LILACS), Banco de Dados em Enfermagem (BDENF) e Serviço de Busca da Biblioteca Nacional de Medicina dos Estados Unidos da América (PUBMED).

Para prover a seleção dos artigos utilizou-se a terminologia padronizada dos Descritores em Ciência da Saúde (DECS). Sendo assim, os descritores utilizados para a pesquisa nas bases de dados MEDLINE, LILACS e BDENF foram: "Estratégia Saúde da Família", "Educação em Enfermagem" e "Gestão em saúde", enquanto que na PUBMED utilizaram-se "Family health strategy", "nurse education" e "health management". Salienta-se o alto grau de semelhança dos descritores da PUBMED com aqueles utilizados nas bases de dados anteriormente citadas.

O percurso metodológico foi operacionalizado por meio das seguintes etapas: elaboração da pergunta norteadora; estabelecimento de descritores; identificação e seleção dos artigos pelos critérios de inclusão/exclusão; caracterização dos estudos; análise e discussão a respeito do processo de formação do 
enfermeiro para o desenvolvimento de habilidades gerenciais na APS e síntese do conhecimento dos artigos analisados $^{(15)}$. Este processo foi ilustrado na Figura 1.

Figura 1: Fluxograma com as etapas do percurso metodológico. Sobral, CE, Brasil, 2017.

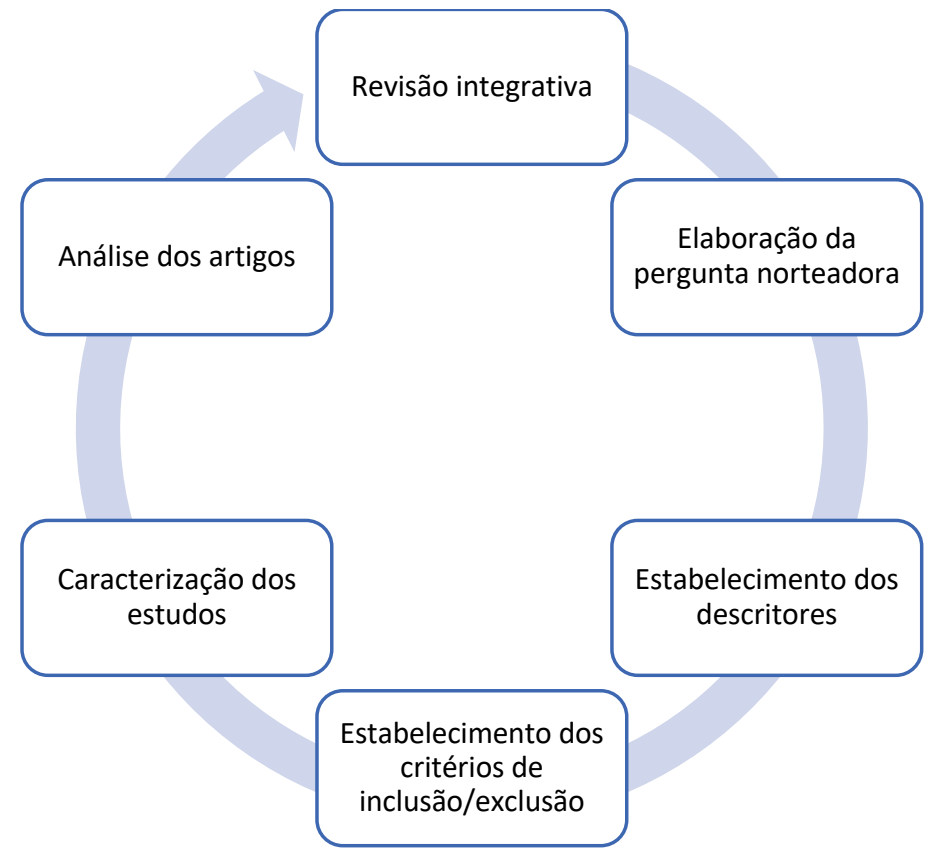

Realizou-se o cruzamento dos três descritores selecionados nas bases MEDLINE, LILACS e BDENF sendo adotado como combinação “Estratégia Saúde da Família” AND “Educação em Enfermagem” AND “Gestão em saúde", obtendo-se uma amostra de 319 artigos. Já em relação à busca na PUBMED, o cruzamento dos descritores "family health strategy, nurse education e health management" gerou um total de 31 artigos. Vale ressaltar que em toda a busca se usou o operador booleano AND entre os descritores nas bases consultadas.

Os critérios de inclusão definidos para a seleção das produções científicas foram: artigos disponíveis na íntegra em formato eletrônico que retratassem a temática estudada nos últimos 10 anos (2007 a 2017) no idioma português e inglês. Como critérios de exclusão foram considerados: documentos técnicos, resumos de congressos, anais, editoriais, revisões da literatura, dissertações e teses de doutorado. Assim, após a utilização dos filtros, resultaram 350 artigos.

Dessa forma, foram pré-selecionados um total de 24 artigos por meio da leitura dos títulos e resumos, os quais foram, posteriormente, submetidos à leitura na íntegra a fim de verificar a adequação aos critérios de inclusão. O resultado desse processo foi ilustrado na Figura 2.

A análise dos achados foi realizada a partir da leitura minuciosa das produções com o intuito de constatar a aderência ao objetivo da presente pesquisa, obtendo-se 14 artigos para análise. Assim, para facilitar a análise do conteúdo das produções foi utilizado um instrumento de coleta de dados (Quadro 1) criado pelos autores com o objetivo de construir um banco de dados de fácil acesso.

Para avaliação crítica dos estudos, identificou-se a classificação do tipo de evidências das pesquisas, classificados como: Nível I. Evidências oriundas de revisão sistemática ou meta-análise de todos relevantes ensaios clínicos randomizados controlados ou provenientes de diretrizes clínicas baseadas em revisões sistemáticas de ensaios clínicos randomizados controlados; Nível II. Evidências derivadas de pelo menos um ensaio clínico 
randomizado controlado bem delineado; Nível III. Evidências obtidas de ensaios clínicos bem delineados sem randomização; Nível IV. Evidências provenientes de estudos de coorte e de caso-controle bem delineados; Nível V. Evidências originárias de revisão sistemática de estudos descritivos e qualitativos; Nível VI. Evidências derivadas de um único estudo descritivo ou qualitativo; Nível VII. Evidências oriundas de opinião de autoridades e/ou relatório de comitês de especialistas ${ }^{(16)}$.

Figura 2: Distribuição dos artigos selecionados nas bases de dados para análise após leitura na íntegra. Sobral, CE, Brasil, 2017.

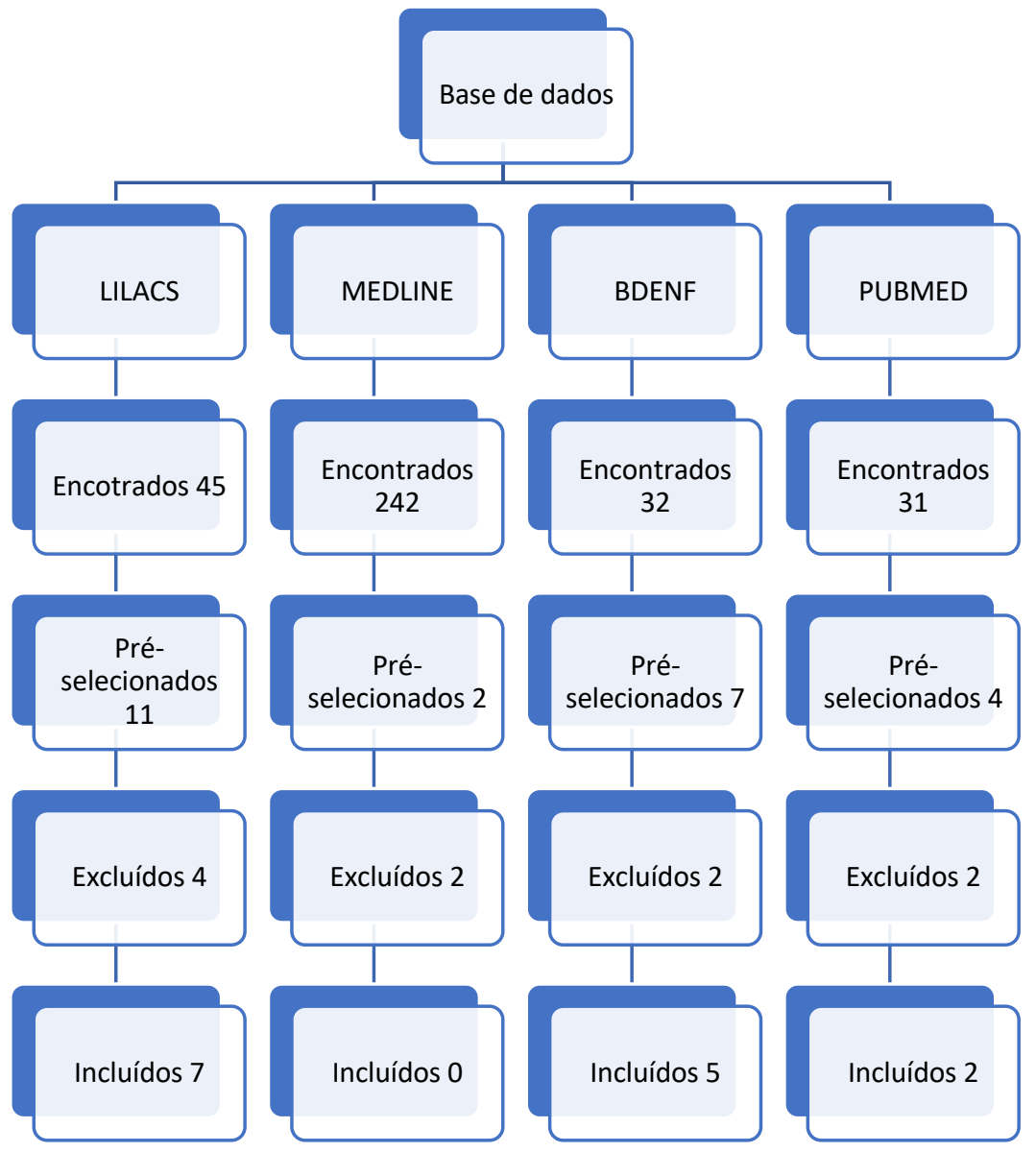

Em seguida, a análise e síntese dos dados extraídos dos artigos foram realizadas de forma descritiva, o que permitiu examinar e classificar os dados. Destacou-se a apresentação dos resultados e sua análise discursiva que objetivaram reunir o conhecimento motivado sobre o tema referido nesta revisão integrativa.

\section{RESULTADOS E DISCUSSÃO}

O Quadro 1 apresenta os 14 artigos elencados contemplando suas características segundo título, nível de evidência, autores, ano, periódico, objetivo, delineamento metodológico, amostra e resultados. 
Quadro 1: Caracterização das produções científicas selecionadas para o estudo, quanto ao título, nível de evidência, autores, ano, periódico, objetivo, delineamento metodológico, amostra e resultados. Sobral, CE, Brasil, 2017.

\begin{tabular}{|c|c|c|c|}
\hline $\begin{array}{l}\text { TíTULO / NÍVEL DE EVIDÊNCIA / AUTORES / } \\
\text { PERIÓDICO / ANO }\end{array}$ & OBJETIVO & $\begin{array}{l}\text { DELINEAMENTO METODOLÓGICO } \\
\text { / AMOSTRA }\end{array}$ & RESULTADOS \\
\hline $\begin{array}{c}\text { Processo de trabalho e competências } \\
\text { gerenciais do enfermeiro da Estratégia saúde } \\
\text { da família(17) / VI / De Paula M, Maris PA, } \\
\text { Elizabeth B, Eduardo EA, Macagi STS / Revista } \\
\text { RENE / (2013) }\end{array}$ & $\begin{array}{l}\text { Identificar as dimensões do processo de } \\
\text { trabalho do enfermeiro em uma unidade da } \\
\text { ESF e correlacionar as competências } \\
\text { necessárias para o desenvolvimento de } \\
\text { atividades gerenciais }\end{array}$ & $\begin{array}{l}\text { Pesquisa descritiva que utilizou a } \\
\text { técnica de observação não } \\
\text { participante durante } 160 \text { horas de } \\
\text { trabalho de quatro enfermeiros }\end{array}$ & $\begin{array}{l}\text { Na dimensão gerencial o enfermeiro exerce funções } \\
\text { administrativas e mobiliza competências gerais e específicas } \\
\text { para a realização das suas atividades. A Educação } \\
\text { Permanente em Saúde (EPS) aparece como competência } \\
\text { gerencial que articula os saberes com os profissionais que } \\
\text { compõem a equipe e os usuários do sistema }\end{array}$ \\
\hline $\begin{array}{l}\text { Coordenação de unidades de saúde da } \\
\text { família por enfermeiros: desafios e } \\
\text { potencialidades (18) / VI / Melo RC, Machado } \\
\text { ME / Revista Gaúcha de Enfermagem / } \\
\text { (2013) }\end{array}$ & $\begin{array}{l}\text { Analisar a coordenação das Unidades de } \\
\text { Saúde da Família (USF) realizada por } \\
\text { enfermeiros }\end{array}$ & $\begin{array}{l}\text { Estudo descritivo com abordagem } \\
\text { qualitativa realizado com oito } \\
\text { enfermeiros da rede de atenção } \\
\text { básica de Porto Alegre/RS }\end{array}$ & $\begin{array}{l}\text { Os resultados demonstraram desafios e qualidades que } \\
\text { compõem o trabalho dos enfermeiros coordenadores. } \\
\text { Como uma das dificuldades a formação acadêmica foi } \\
\text { apontada como insuficiente para dar conta da coordenação } \\
\text { de uma USF }\end{array}$ \\
\hline $\begin{array}{l}\text { O planejamento estratégico situacional no } \\
\text { ensino da gestão em saúde da família(19) / VII } \\
\text { / Kleba ME, Krauser IM, Vendruscolo C / } \\
\text { Texto e contexto Enfermagem / (2011) }\end{array}$ & $\begin{array}{l}\text { Analisar a aplicação do planejamento } \\
\text { estratégico situacional (PES) como ferramenta } \\
\text { de gestão na saúde coletiva }\end{array}$ & $\begin{array}{l}\text { Relato de experiência a partir de } \\
\text { reflexões sobre a implementação } \\
\text { das etapas do planejamento } \\
\text { estratégico situacional nas } \\
\text { unidades selecionadas }\end{array}$ & $\begin{array}{l}\text { O exercício do PES permitiu aos estudantes desenvolver } \\
\text { competências de comunicação, liderança, tomada de } \\
\text { decisões e administração e gerenciamento, além de } \\
\text { promover maior aproximação entre atores do ensino e do } \\
\text { serviço }\end{array}$ \\
\hline $\begin{array}{c}\text { A função gerencial do enfermeiro na } \\
\text { Estratégia Saúde da Família: limites e } \\
\text { possibilidades }{ }^{(20)} \text { / VI / Jonas LT, Rodrigues } \\
\text { HC, Resck ZMR / Revista de Atenção Primária } \\
\text { à Saúde / (2011) }\end{array}$ & $\begin{array}{l}\text { Compreender e analisar os limites e as } \\
\text { possibilidades do processo de trabalho } \\
\text { gerencial do enfermeiro na equipe do } \\
\text { Programa Saúde da Família }\end{array}$ & $\begin{array}{l}\text { Pesquisa qualitativa com } \\
\text { participação dos profissionais de } \\
\text { duas equipes de Saúde da Família } \\
\text { do município de Alfenas-MG, } \\
\text { totalizando } 15 \text { sujeitos }\end{array}$ & $\begin{array}{l}\text { O enfermeiro assume o papel de gerente, coordenando o } \\
\text { trabalho das Unidades de Saúde da Família e dos membros } \\
\text { da equipe, olhando não só para o trabalho da Enfermagem } \\
\text { e dos Agentes Comunitários de Saúde, mas ampliando este } \\
\text { olhar para toda equipe multiprofissional. }\end{array}$ \\
\hline $\begin{array}{l}\text { Educação permanente em saúde: } \\
\text { construções de enfermeiros da Estratégia } \\
\text { Saúde da Família(21) / VI / Santos AR, } \\
\text { Coutinho ML / Revista Baiana de Saúde } \\
\text { Pública / (2014) }\end{array}$ & $\begin{array}{l}\text { Analisar a participação dos enfermeiros das } \\
\text { Unidades de Saúde da Família de Itajuípe, } \\
\text { Bahia, nas ações de Educação Permanente em } \\
\text { Saúde desenvolvidas no município. }\end{array}$ & $\begin{array}{l}\text { Estudo qualitativo. Participaram } \\
\text { oito enfermeiros, sendo dois deles } \\
\text { atuantes em programas } \\
\text { específicos na Unidade Básica de } \\
\text { Saúde e os demais são } \\
\text { responsáveis pelas USF }\end{array}$ & $\begin{array}{l}\text { Os resultados apontam para uma grande associação por } \\
\text { parte dos profissionais de saúde da EPS com os processos } \\
\text { de educação voltados para as necessidades da população e } \\
\text { não para o universo do processo de trabalho em saúde }\end{array}$ \\
\hline $\begin{array}{c}\text { Sistema Único de Saúde e da Família na } \\
\text { formação acadêmica do enfermeiro(22) / VII / } \\
\text { Costa RKS, Miranda FAN / Revista Brasileira } \\
\text { de Enfermagem / (2009) }\end{array}$ & $\begin{array}{l}\text { Refletir sobre os movimentos de mudança na } \\
\text { graduação em Enfermagem, enfatizando a } \\
\text { preparação profissional para atender a atual } \\
\text { conformação do setor saúde, a partir da } \\
\text { consolidação do SUS/ESF. }\end{array}$ & Estudo Reflexivo & $\begin{array}{l}\text { A formação acadêmica do enfermeiro sofreu modificações } \\
\text { ao longo dos anos com a nova realidade sócio-sanitária do } \\
\text { País. Porém, há a necessidade de mudanças efetivas nos } \\
\text { currículos como forma de intervir na reorganização do } \\
\text { modelo assistencial }\end{array}$ \\
\hline
\end{tabular}


Quadro 1: Caracterização das produções científicas selecionadas para o estudo, quanto ao título, nível de evidência, autores, ano, periódico, objetivo, delineamento metodológico, amostra e resultados. Sobral, CE, Brasil, 2017.

\begin{tabular}{|c|c|c|c|}
\hline $\begin{array}{l}\text { TíTULO / NÍVEL DE EVIDÊNCIA / AUTORES / } \\
\text { PERIÓDICO / ANO }\end{array}$ & OBJETIVO & $\begin{array}{l}\text { DELINEAMENTO METODOLÓGICO } \\
\text { / AMOSTRA }\end{array}$ & RESULTADOS \\
\hline $\begin{array}{l}\text { A prática gerencial do enfermeiro no PSF na } \\
\text { perspectiva da sua ação pedagógica } \\
\text { educativa: uma breve reflexão(23) / VI / Bôas } \\
\text { LMFMV, Araújo MBS, Timóteo RPS / Ciência } \\
\text { e Saúde Coletiva / (2008) }\end{array}$ & $\begin{array}{l}\text { Contribuir com a reflexão acerca do fazer } \\
\text { gerencial do enfermeiro no PSF, articulando a } \\
\text { ação pedagógica/educacional. }\end{array}$ & Estudo qualitativo reflexivo. & $\begin{array}{l}\text { O enfermeiro na ESF desenvolve atividades relacionadas aos } \\
\text { campos de assistência, gerência, assim como formação. É } \\
\text { em uma perspectiva multidimensional que deve ser } \\
\text { entendida as atribuições, ações do profissional de } \\
\text { Enfermagem, sendo necessário uma visão mais ampla, que } \\
\text { seja capaz de atender aos novos arranjos e desafios no SUS. }\end{array}$ \\
\hline $\begin{array}{l}\text { Gestão do trabalho em unidades básicas de } \\
\text { saúde(8) / VI / Celedônio RM, Fé MCM, } \\
\text { Mendes AHL, Mendes AHL, Chaves TLF / } \\
\text { Revista de Enfermagem da UFPE / (2017) }\end{array}$ & $\begin{array}{l}\text { Analisar a gestão do processo de trabalho dos } \\
\text { gerentes das Unidades Básicas de Saúde }\end{array}$ & $\begin{array}{l}\text { Estudo descritivo-exploratório, de } \\
\text { abordagem qualitativa realizado } \\
\text { em oito Centros de Saúde da } \\
\text { Família, com oito enfermeiros }\end{array}$ & $\begin{array}{l}\text { Os enfermeiros apresentaram várias atividades na UBS além } \\
\text { do gerenciamento, tendo sobrecarga de trabalho, o que } \\
\text { pode gerar tanto insatisfação por parte dos profissionais } \\
\text { como prejudicar o serviço assistencial. }\end{array}$ \\
\hline $\begin{array}{l}\text { Exercício da liderança do enfermeiro na } \\
\text { Estratégia Saúde da Família(24) / VI / Lima FS, } \\
\text { Amestosy SC, Jacondino MB, Trindade LM, } \\
\text { Silva CN, Fuculo Junior PRB / Revista de } \\
\text { Pesquisa: Cuidado é fundamental / (2016) }\end{array}$ & $\begin{array}{l}\text { Conhecer o exercício da liderança do } \\
\text { enfermeiro na ESF, bem como as dificuldades } \\
\text { e estratégias adotadas pelos enfermeiros para } \\
\text { liderar. }\end{array}$ & $\begin{array}{l}\text { Estudo qualitativo, descritivo e } \\
\text { exploratório realizado com } 12 \\
\text { enfermeiros da ESF de Pelotas, RS }\end{array}$ & $\begin{array}{l}\text { Evidenciou-se que o enfermeiro, enquanto líder é visto } \\
\text { como um exemplo a ser seguido, responsável por contribuir } \\
\text { com a motivação da equipe. }\end{array}$ \\
\hline $\begin{array}{l}\text { O uso de indicadores como ferramenta de } \\
\text { gestão na estratégia saúde da família(25) / VI / } \\
\text { Paes LG, Bellato TMS, Machado BP, Lima SBS } \\
\text { / Revista de Enfermagem da UFSM / (2015) }\end{array}$ & $\begin{array}{c}\text { Verificar o uso dos indicadores de saúde no } \\
\text { cotidiano do trabalho de enfermeiros } \\
\text { gerentes da ESF, em um município de Santa } \\
\text { Catarina, Brasil. }\end{array}$ & $\begin{array}{c}\text { Pesquisa descritiva com } \\
\text { abordagem qualitativa realizada } \\
\text { com treze enfermeiros gerentesda } \\
\text { ESF }\end{array}$ & $\begin{array}{c}\text { Os indicadores são concebidos como uma possibilidade } \\
\text { para o planejamento e avaliação das ações em saúde na } \\
\text { ESF, embora tenha se evidenciado certo distanciamento } \\
\text { entre a teoria e prática. }\end{array}$ \\
\hline $\begin{array}{l}\text { Estilos de liderança dos enfermeiros que } \\
\text { atuam na estratégia de saúde da família(26) / } \\
\text { VII / Costa SEM, Peres AM, Bernardino E, } \\
\text { Sade PMC / Revista Ciência, Cuidado e Saúde } \\
\text { / (2015) }\end{array}$ & $\begin{array}{l}\text { Relacionar as atividades desenvolvidas pelos } \\
\text { enfermeiros que atuam em Unidades Básicas } \\
\text { de Saúde em um município do Sul do Brasil } \\
\text { com os principais estilos de liderança } \\
\text { situacional propostos pelo modelo de Hersey } \\
\text { e Blanchard. }\end{array}$ & $\begin{array}{l}\text { Pesquisa quantitativa e } \\
\text { exploratória com nove unidades } \\
\text { com } 17 \text { enfermeiros }\end{array}$ & $\begin{array}{l}\text { Os resultados da pesquisa apontaram predomínio no estilo } \\
\text { de liderança "Determinar", de forma que os enfermeiros } \\
\text { utilizam mais frequentemente uma liderança diretiva, em } \\
\text { que se destaca uma supervisão mais atenta sobre os } \\
\text { liderados, com nível de maturidade de baixo a moderado, } \\
\text { que requer destes o desenvolvimento das atividades a } \\
\text { partir de orientações específicas dadas pelo líder. }\end{array}$ \\
\hline $\begin{array}{l}\text { Enfermeiros coordenadores de equipe do } \\
\text { programa saúde da família: perfil } \\
\text { profissional(27) / VI / Rocha BS, Munari DB, } \\
\text { Bezerra ALQ, Melo LKA / Revista de } \\
\text { Enfermagem da UERJ / (2009) }\end{array}$ & $\begin{array}{l}\text { Caracterizar o perfil profissional dos } \\
\text { enfermeiros do PSF que atuam na } \\
\text { coordenação da equipe. }\end{array}$ & $\begin{array}{c}\text { Estudo descritivo de abordagem } \\
\text { quantitativa realizado com } 94 \\
\text { enfermeiros do PSF }\end{array}$ & $\begin{array}{l}\text { Os resultados mostraram que maioria deles é mulher } \\
(97,9 \%) ; \text { que raramente realizam territorialização e } \\
\text { mapeamento em suas áreas de abrangência e que os } \\
\text { profissionais sob sua supervisão raramente realizam } \\
\text { atividades de educação em saúde por iniciativa própria. }\end{array}$ \\
\hline
\end{tabular}


Quadro 1: Caracterização das produções científicas selecionadas para o estudo, quanto ao título, nível de evidência, autores, ano, periódico, objetivo, delineamento metodológico, amostra e resultados. Sobral, CE, Brasil, 2017.

\begin{tabular}{|c|c|c|c|}
\hline $\begin{array}{l}\text { TÍTULO / NÍVEL DE EVIDÊNCIA / AUTORES / } \\
\text { PERIÓDICO / ANO }\end{array}$ & OBJETIVO & $\begin{array}{l}\text { DELINEAMENTO METODOLÓGICO } \\
\text { / AMOSTRA }\end{array}$ & RESULTADOS \\
\hline $\begin{array}{c}\text { Evaluation of an interactive web-based } \\
\text { nursing course with streaming videos for } \\
\text { medication administration skills(28) / VI / } \\
\text { Sowana AK, Idhailba JA / Rev. Families, } \\
\text { Systems \& Health / (2014) }\end{array}$ & $\begin{array}{l}\text { Relatar uma experiência de estudantes de } \\
\text { enfermagem com novas estratégias de ensino } \\
\text { em Enfermagem. }\end{array}$ & Pesquisa qualitativa, estudo misto & $\begin{array}{l}\text { Evidenciou-se uma deficiência do ensino no curso de } \\
\text { Enfermagem, incluindo a incapacidade dos alunos para } \\
\text { compreenderem os procedimentos como um todo e } \\
\text { currículo fragmentado, impedindo uma aprendizagem } \\
\text { baseada em problemas. }\end{array}$ \\
\hline $\begin{array}{l}\text { The participant's perspective: learning from } \\
\text { an aggression management training course } \\
\text { for nurses. Insights from a qualitative } \\
\text { interview study(29) / IV / Birgit HRN, } \\
\text { Breimaier HERN, Halfens RJG, Jos MGA, Hahn } \\
\text { S / Scand J CaringSci / (2016) }\end{array}$ & $\begin{array}{l}\text { Explorar e descrever, do ponto de vista da } \\
\text { enfermeira o aprendizado obtido com o } \\
\text { atendimento ao gerenciamento de agressões. }\end{array}$ & Estudo qualitativo descritivo & $\begin{array}{l}\text { Na prática os enfermeiros são continuamente desafiados a } \\
\text { lidarem com o impacto emocional, sendo importante } \\
\text { fortalecer a capacidade de gerir situações, promovendo } \\
\text { habilidades de enfrentamento e resiliência. }\end{array}$ \\
\hline
\end{tabular}


Embora nem todos os artigos levantados e estudados possam ser considerados efetivamente acerca da formação do enfermeiro para habilidades gerenciais no âmbito da ESF, os resultados evidenciaram também importantes e diferentes aspectos entre si nos modos das práticas de gestão deste profissional nos serviços de saúde.

A partir desta evidência, julgou-se necessário, para este estudo, apresentar uma categoria sobre o processo de trabalho do enfermeiro gestor antes de reunir as produções que enfatizam, de forma mais direta, a formação do enfermeiro para o desenvolvimento de habilidades gerenciais.

Assim, para discutir o tema de forma mais fundamentada, identificamos três categorias temáticas, a saber: "Processo de trabalho do enfermeiro gestor: ferramenta potente para operar nas práticas de saúde", "Educação Permanente em Saúde como estratégia de qualificação da prática gerencial do enfermeiro" e “Atividades gerenciais em Enfermagem: reflexos dos processos formativos", as quais serão abordadas e discutidas a seguir.

\section{Processo de trabalho do enfermeiro gestor: ferramenta potente para operar nas práticas de saúde}

Como dito, esta categoria apresenta-se como extra pelo fato de julgarmos pertinente abordar os desafios e potencialidades do trabalho do enfermeiro gestor, para dar maior sustentação às discussões fomentadas na terceira categoria referente aos processos formativos e por estes aspectos terem se destacado de forma significativa nas produções analisadas.

No Brasil, as atividades da dimensão gerencial possuem destaque no processo de trabalho do enfermeiro e visam garantir a qualidade da assistência de Enfermagem, assim como o bom funcionamento das Unidades de Saúde da Família(17).

Pesquisadores apontam a atividade gerencial como uma prática dinâmica, complexa e que requer habilidades com capacidade de análise crítica e de tomada de decisão, além da capacidade de diálogo, visando estabelecer vínculos tanto entre os membros da equipe, quanto entre os agentes do cuidado e os usuários dos serviços $^{(30)}$.

Nesse contexto, a atuação do enfermeiro como gestor está se tornando uma atividade muito frequente, sendo esta regulamentada pelo Conselho Federal de Enfermagem, o qual estabelece atribuições de chefia, planejamento, organização, coordenação e avaliação dos serviços de Enfermagem como um dos principais instrumentos para o exercício gerencial do enfermeiro ${ }^{(8)}$.

Dessa forma, entre tais práticas de gestão, autores descrevem o planejamento como essencial para o desenvolvimento de uma ação, as quais serão realizadas por enfermeiros na rotina dos serviços de saúde com ênfase na ideia de que estes profissionais possam realizar simultaneamente atividades de cuidado e gerenciamento $^{(31)}$.

Assim, pesquisadores revelam que o enfermeiro é reconhecido como o profissional que mais detém informações sobre o funcionamento dos serviços, sendo constantemente requisitado para esclarecer e resolver situações no decorrer da assistência( ${ }^{(32)}$. Então, como uma de suas prerrogativas profissionais, o gerenciamento da equipe e dos serviços de Enfermagem têm aproximado o enfermeiro do papel de gerenciar unidades de saúde.

Corroborando com tais assertivas, um estudo recente mostra a unanimidade de enfermeiros exercendo cargos de gerente e coordenador nos oito Centros de Saúde da Família analisados pela pesquisa, constituindo-se 
como um agente articulador das ações desenvolvidas pela equipe e organizador do cuidado ${ }^{(8)}$. Outro estudo revela que das 108 UBS estudadas no estado do Paraná, 107 eram gerenciadas por enfermeiros ${ }^{(9)}$.

Autores relacionam este aspecto com o fato de que os mesmos tenham uma grande apropriação dos saberes e práticas nos campos da Saúde Pública e da Saúde Coletiva, em especial da APS. Outra questão determinante a ser considerada é o longo tempo de atuação dos enfermeiros em um mesmo território, proporcionando um amplo conhecimento deste e o estabelecimento de vínculo com a comunidade. Além disso, um terceiro aspecto provavelmente se justifique pelo baixo valor pago pelo cargo de gerente, sendo o mercado de trabalho em Enfermagem cerceado por uma política de baixos salários e isso, talvez, gere a maior adesão de enfermeiros ao cargo em relação a outras profissões da saúde ${ }^{(15)}$.

A legislação que regulamenta a Política Nacional da Atenção Básica (PNAB) ${ }^{(33)}$ não apresenta, como competência do enfermeiro, o gerenciamento da unidade, mas atribui a ele a participação dos processos gerenciais para o funcionamento desta, enquanto que os participantes de um estudo sobre a função gerencial do enfermeiro atribuem a este profissional o exercício de funções de gerente do serviço.

Mesmo com a revisão e mudanças nas diretrizes da nova PNAB, as atribuições do enfermeiro na APS permanecem, como o ato de planejar, gerenciar e avaliar as ações desenvolvidas pelos técnicos/auxiliares de Enfermagem, Agente Comunitário de Saúde e Agente Comunitário de Endemias em conjunto com os outros membros da equipe, mas não destinam os enfermeiros como os responsáveis gerenciais da unidade ${ }^{(34)}$.

Portanto, é necessário aproveitar essa condição para se posicionar e conquistar maior espaço no mercado de trabalho, além de caminhar em busca do reconhecimento e valorização profissional, principalmente no nível da APS, em que os espaços de atuação têm-se consolidado para o enfermeiro e crescido progressivamente ${ }^{(20)}$.

Diante disso, o grande desafio dos enfermeiros gestores é a necessidade de rever sua prática diante de novos paradigmas, sendo indispensável repensar os processos de trabalho, bem como adotar metodologias, instrumentos e conhecimentos diferentes dos atualmente instituídos ${ }^{(23)}$.

Para isso, um estudo sugere que a gestão desses processos deve ser compartilhada, uma cogestão, propiciando a construção dos projetos de forma participativa, a fim de não sobrecarregar o trabalho do enfermeiro. Nessa perspectiva, este estudo reflete sobre estes aspectos que perpassam o trabalho deste profissional na ESF, pois a partir da compreensão de suas reais atribuições e potencializando a participação dos membros da equipe, o enfermeiro poderá minimizar sua sobrecarga de trabalho e destinar esforços para fortalecer a atenção à saúde ${ }^{(24)}$.

Esta afirmação corrobora com um estudo de revisão sobre as práticas de Enfermagem no contexto da APS, o qual mostra que os enfermeiros desenvolvem ações de cuidado e gerenciamento, muitas vezes simultaneamente, para que sejam vistos como indivíduos polivalentes na equipe. No entanto, os autores sugerem que as ações de gestão devem ser conjuntamente realizadas por toda a equipe de saúde para que os enfermeiros não realizem atividades que são uma responsabilidade de outros profissionais ${ }^{(31)}$.

Nessa perspectiva, ao coordenar a equipe da ESF, o enfermeiro tem a atribuição de incentivar o trabalho coletivo para efetivar o trabalho em equipe, atingir a produtividade máxima e um nível de qualidade de serviço em saúde. Porém, nem sempre é considerado habilidade e formação acadêmica adequada para exercer essa função(27). 
Dessa forma, pesquisadores revelam que a falta de formação específica pode ser considerada uma dificuldade para o gerenciamento, principalmente no que concerne ao conhecimento com relação ao processo decisório e que o desenvolvimento gerencial, definido a partir de um modelo assistencial centrado no cuidado, contribui na redefinição do papel gerencial, necessitando, porém, da adoção de uma abordagem dialética durante a análise das organizações ${ }^{(30)}$.

Diante desse desafio, um estudo menciona a Educação Permanente em Saúde (EPS) como uma estratégia inovadora de promover a qualificação dos profissionais de saúde de forma a suprir as deficiências e lacunas existentes na formação no que concerne aos processos gerenciais dos enfermeiros ${ }^{(21)}$. Assim, o papel do enfermeiro gestor deve ser promovido e fortalecido não apenas na formação de forma exclusiva, mas como processo de educação permanente no cotidiano, a partir das demandas da vida profissional.

\section{Educação Permanente em Saúde como estratégia de qualificação da prática gerencial do enfermeiro}

Esta categoria surgiu diante da evidência constatada, nos estudos selecionados, da necessidade imediata de investimentos na qualificação das equipes e dos gestores, onde os estudos apontam a EPS como eixo estratégico para o fortalecimento dos mecanismos de gestão e controle social orientado pelo compromisso com a saúde dos usuários ${ }^{(17,21,23-25)}$.

Nesse contexto, pesquisadores enfatizam que a EPS se apresenta como uma proposta de ação estratégica capaz de contribuir para a transformação dos processos formativos, das práticas pedagógicas e de saúde e para a organização dos serviços, empreendendo um trabalho articulado entre o sistema de saúde, em suas várias esferas de gestão, e as instituições formadoras ${ }^{(35)}$.

Nessa perspectiva, estudiosos mostram que a debilidade dos currículos da graduação no que concerne à atuação na APS tem motivado os profissionais enfermeiros a buscarem especialização múltipla e isto tem fomentado um dilema de identidade, levando os diversos profissionais a uma indefinição de papéis, influenciados pela formação de especialista, além do trabalho fragmentado e precarizado(32).

Diante desta fragilidade, alguns autores defendem o uso de novas estratégias de ensino que venham potencializar a aprendizagem do estudante de Enfermagem, sendo atribuído uma ênfase à mudança do papel dos alunos para se tornarem mais independentes, de forma a ressaltar o ensino inovador como estratégia fundamental para a transformação da educação(28).

Uma pesquisa aponta que os enfermeiros, em seus espaços de trabalho, sentem a necessidade de uma educação permanente por, muitas vezes, encontrarem dificuldades e limitações nas atividades cotidianas e por necessitarem de atualizações periódicas para uma prática efetiva, com maior resolubilidade e compartilhamento entre os coletivos de trabalho. Assim, pode-se afirmar também que a EPS é a proposta mais apropriada para trabalhar a construção do novo modo de operar o sistema de saúde brasileiro, uma vez que permite articular gestão, atenção e formação para o enfrentamento dos problemas de cada equipe de saúde ${ }^{(21)}$.

Dessa forma, autores afirmam que a EPS favorece a Enfermagem pelas características de seus instrumentos básicos, visto que sua prática necessita de domínios e competências, os quais se baseiam na comunicação, na observação, na aplicação de princípios científicos, na destreza manual, no planejamento, na avaliação, no trabalho em equipe e na utilização de recursos da comunidade ${ }^{(36-37)}$. 
Dada a importância da EPS para a reorientação do modelo de atenção à saúde e da ESF para a consolidação do SUS, é mister o conhecimento acerca das maneiras que os enfermeiros têm usado essa ferramenta para a melhoria de seu processo de trabalho nas unidades, bem como para a reorientação do modelo de atenção e gestão $^{(21)}$.

Então, a partir da leitura das produções científicas selecionadas para esta revisão, fica evidente a necessidade e importância da EPS como ferramenta que possibilita melhorias no processo de trabalho, de forma a oferecer uma dimensão substancial para caracterizar tal relevância, mas vale aqui ressaltar o cuidado que os profissionais e gestores devem ter em associar a EPS com atividades de caráter meramente atualizador, desconsiderando, uma das prerrogativas da EPS, que é a problematização da realidade vivenciada pelas equipes de saúde.

A partir do exposto nesta categoria, é imprescindível que na atualidade, gestores e gerentes estejam inseridos num arrojado processo de EPS para que possam administrar, com competência, todos os processos que compreendam a gestão do cuidado em saúde com a finalidade de garantir uma prática mais integral, autônoma e resolutiva para o exercício de uma gerência efetiva frente às suas ações e responsabilidades ${ }^{(37)}$.

\section{Atividades gerenciais em Enfermagem: reflexos dos processos formativos}

Foi possível inferir, de acordo com a literatura consultada que, para realizar um bom gerenciamento, o enfermeiro tem de aproximar-se da essência de seu trabalho, o cuidado, e compreender seu processo como um conjunto de ações voltadas para o atendimento das necessidades de saúde dos usuários que engloba todas as atividades assistenciais e gerenciais. Assim, entendeu-se que o enfermeiro precisa ter, em sua formação, os enfoques articulados, para que não ocorra cisão das atividades assistenciais e gerenciais na prática profissional(20).

Porém, como foi visto nas categorias anteriores, a educação dos profissionais de saúde tem sido criticada por não estar voltada às necessidades de saúde da sociedade. A maioria dos profissionais tem uma formação densa no campo biomédico e clínico, mas pequena formação em Ciências Sociais e Humanas, o que reduz suas habilidades em manejar questões políticas, sociais, culturais e econômicas, exigidas pela gerência(22).

Autores corroboram quando revelam que as teorias administrativas, historicamente mais conhecidas e cujos conhecimentos estão presentes no cotidiano da Enfermagem são utilizadas de forma pouco crítica e reflexiva, determinando, frequentemente, ações reducionistas. Entre as principais características desses modelos destacam-se a fragmentação do trabalho com separação entre concepção e execução, o controle gerencial do processo de produção associado à rígida hierarquia, a racionalização da estrutura administrativa, a impessoalidade nas relações interpessoais e a ênfase em sistemas de procedimentos e rotinas ${ }^{(10)}$.

Nesse contexto, são escassas as oportunidades de aprender como direcionar os poderes sociais, econômicos e políticos que interferem no setor saúde. Além disso, os centros formadores parecem estar distantes dos espaços de tomada de decisões acerca da provisão dos serviços e das políticas de saúde ${ }^{(30)}$.

Estudos realizados no Brasil discutem essa questão e evidenciam a limitação dos enfermeiros coordenadores da ESF no que diz respeito à formação específica para assumir atividades gerenciais, além de desafios deste profissional por se tratar de um papel ainda em construção. Diante disso, a formação em 
Enfermagem deve buscar sua reordenação, promovendo a formação de profissionais que dialoguem com as rápidas transformações da sociedade contemporânea ${ }^{(18)}$.

Mesmo com limitações referentes à formação no campo da gestão, a equipe de saúde compreende o enfermeiro como um elemento significativo na rede de relações e interações, sendo visualizado como mediador e como uma liderança. Ainda, os enfermeiros da ESF desempenham uma atuação importante junto à equipe de saúde, disponibilizando suporte clínico no planejamento do cuidado e gerencial da unidade de saúde ao contribuir para o estabelecimento de boas relações, direcionar as atividades da equipe e conduzir o fluxo de informações ${ }^{(24)}$.

Neste sentido, autores também corroboram quando revelam que os profissionais de Enfermagem são perpetuamente desafiados a lidarem com o impacto emocional, sendo importante fortalecer a capacidade de gerir diversas situações, promovendo habilidades de enfrentamento e resiliência ${ }^{(29)}$.

Estudiosos reiteram que tal fato se dá pelo diferencial que o enfermeiro tem em relação às demais profissões de saúde: que é o conhecimento de administração em sua formação de aproximadamente $25 \%$ dos conteúdos ministrados durante o curso(20).

Assim, as competências específicas da dimensão gerencial do trabalho do enfermeiro se constroem durante a formação, conforme as Diretrizes Curriculares Nacionais exigem, e são desenvolvidas nas experiências cotidianas $^{(30)}$. Com efeito, a formação e a prática profissional do enfermeiro devem transitar entre as dimensões do processo de trabalho, para construir caminhos no desenvolvimento de competências, com maior interação ensino e serviço ${ }^{(5,16)}$.

Nessa perspectiva, estudos apontam para a necessidade de as instituições de ensino definirem um Projeto Político-Pedagógico (PPP), no qual estejam envolvidos universidades, serviço, comunidade, o que vem sendo fortalecido pela política interministerial que visa a mudança na formação em saúde, em todos os níveis. Esta deve ter como premissa a formação de profissionais com perfil necessário para o atendimento às demandas e às necessidades de saúde da população ${ }^{(23)}$.

Com este propósito, autores relatam uma experiência de planejamento estratégico situacional, a qual estabeleceu, em seu PPP, a gestão como um dos eixos condutores do curso ao lado da promoção da saúde e do cuidado holístico. Neste sentido, conteúdos de gestão são trabalhados ao longo do curso como temas transversais das práticas de cuidado, de forma a promover no estudante o reconhecimento do planejamento como componente essencial do cuidado em saúde, bem como de seu papel na liderança como membro da equipe multiprofissional. O PPP do curso salienta a relevância do domínio dos instrumentos da gestão para consolidar a autonomia da profissão, capaz de dialogar com outros trabalhadores e interferir nos rumos da saúde nas várias instâncias ${ }^{(19)}$.

A literatura é vasta ao sinalizar a necessidade de contínua atualização dos processos de gerência empregados no ensino e formação em Enfermagem. A forma de organização do trabalho em saúde deve superar a lógica da produção sistemática e pouco reflexiva, ou seja, a superação do modelo Taylorista de produção e incluir, de forma criativa, a triangulação das teorias na coprodução do trabalho em saúde ${ }^{(18,30)}$.

Diante dessa premissa, um estudo sugere o investimento em pesquisas que indiquem as limitações e dificuldades pelas quais passa o enfermeiro coordenador de equipes na ESF, principalmente considerando as diferenças regionais, além da aplicação de estratégias de ensino inovadoras que possam desenvolver o enfermeiro 
gerente e melhorar sua atuação no que tange às habilidades de administração. Esse conhecimento pode agregar um conjunto de diretrizes para guiar o processo de formação dos enfermeiros para que sejam, de fato, agentes de mudança e consolidação da política nacional de saúde ${ }^{(23)}$.

\section{CONSIDERAÇÕES FINAIS}

Diante do exposto, é possível afirmar que há evidências científicas que enfatizam a importância da dimensão gerencial dentro do processo de formação do enfermeiro. Contudo, verifica-se que os profissionais têm uma formação densa no campo biomédico, mas ainda incipiente em Ciências Sociais, Relações Humanas, Burocráticas e Comportamentais, abrindo lacunas para o exercício de uma gerência efetiva.

Mesmo com tais debilidades na formação, o estudo permitiu inferir que o enfermeiro é considerado o profissional mais direcionado para exercer cargos gerenciais e, a partir disso, os mesmos emanam a necessidade de busca de aperfeiçoamento no campo da gestão.

Logo, essa procura por capacitações, especializações e estudos para desenvolvimento de competências como gerente é verificado quando os enfermeiros já ocupam cargo de gestão por meio, muitas vezes, da EPS, o que deveria ser uma continuidade e complementaridade da formação do profissional, e não um processo formador de enfermeiros gestores.

Dessa forma, se faz relevante pensar que a lógica da formação em Enfermagem deve estar pautada na produção do trabalho gerencial de forma crítica-reflexiva integrando e relacionando as teorias de ensino em saúde com o fazer profissional nos campos de prática, de forma transversal, ao longo de toda a graduação em Enfermagem. Além disso, percebe-se a importância de introduzir e estimular o uso de estratégias de ensino que potencialize o papel do estudante para uma prática mais independente, de forma a promover a aquisição de empoderamento gerencial em busca de um fazer mais eficaz, resolutivo e eficiente.

Assim, o produto deste processo formativo resultará em um profissional com habilidades de gestão capaz de suprir as atuais demandas de saúde da população de acordo com as rápidas transformações epidemiológicas e demográficas que vivenciamos neste País.

Por fim, diante de uma literatura vasta acerca das competências gerenciais e formação neste contexto, torna-se fundamental a progressão contínua de pesquisas nesta área para fornecer apoio aos centros formadores no reconhecimento e identificação dos desafios e fatores limitantes, a fim de que estes sejam enfrentados e superados para incorporação de novas estratégias de formação que potencialize o enfermeiro gestor, a partir da articulação indissociável entre gerenciamento e assistência na APS.

\section{REFERÊNCIAS}

1. Lima DC, Pereira AA, Drummond ES. Percepção dos acadêmicos de odontologia da UNIFAL/MG quanto à formação em Saúde da Família. Rev Odontol Bras Central [Internet]. 2013 [acesso em: 28 nov. 2018];21(60):50-5.Disponível em: http://www.robrac.org.br/seer/index.php/ROBRAC/article/view/670.

2. Nunes EFPA, Carvalho BG, Nociletto SCS, Cordoni Junior L. Trabalho gerencial em Unidades Básicas de Saúde de municípios de pequeno porte no Paraná, Brasil. Interface (Botucatu) [Internet]. 2016 [acesso em: 28 nov. 2018];20(58):57384. Disponível em: https://doi.org/10.1590/1807-57622015.0065. 
3. Araújo NP, Miranda TOS, Garcia CPC. O estado da arte sobre a formação do enfermeiro para a gestão em saúde. Revista Enfermagem Contemporânea [Internet]. 2014 [acesso em: 28 nov. 2018];3(2):165-80. Disponível em:

https://www5.bahiana.edu.br/index.php/enfermagem/article/view/365.

4. Trevisan DD, Minzon DT, Testi CV, Ramos NA, Carmona EV, Silva EM. Formação de enfermeiros: distanciamento entre a graduação e a prática profissional. Ciênc. cuid. saúde [Internet]. 2013 [acesso em: 28 nov. 2018];12(2):331-7. Disponível em: https://doi.org/10.4025/cienccuidsaude.v12i2.19643.

5. Ángel Jimenez GM, Lopera Arrubla CP. Relevancia y nivel de aplicación de las competencias de gestión en enfermería. Invest Educ Enferm [Internet]. 2013 [acesso em: 28 nov. 2018];31(1):9-19. Disponível em:

http://www.scielo.org.co/scielo.php?pid=S0120-53072013000100002\&script=sci_arttext\&tlng=es.

6. Regis CG, Batista NA. O enfermeiro na área da saúde coletiva: concepções e competências. Rev Bras Enferm [Internet]. 2015 [acesso em: 28 nov. 2018];68(5):830-6. Disponível em: https://doi.org/10.1590/0034-7167.2015680510i.

7. Resolução CNE/CES no 3, de 7 de novembro de 2001 (BR) [Internet]. Institui Diretrizes Curriculares Nacionais do Curso de Graduação em Enfermagem. Diário Oficial da União. 09 nov. 2001 [acesso em: 28 nov. 2018]. Disponível em:

http://portal.mec.gov.br/cne/arquivos/pdf/CESO3.pdf.

8. Celedônio RM, Fé MCM, Mendes AHL, Mendes AHL, Chaves TLF. Gestão do trabalho em unidades básicas de saúde. Revista de Enfermagem UFPE Online [Internet]. 2017 [acesso em: 28 nov. 2018];11(Supl.1):341-50. Disponível em: https://periodicos.ufpe.br/revistas/revistaenfermagem/article/view/11914.

9. Carvalho BG, Peduzzi M, Nunes EFPA, Leite FS, Silva JAM. Management of basic health units in municipalities of different size: profile and management instruments. Rev Esc Enferm USP [Internet]. 2014 [acesso em: 28 nov. 2018];48(5):907-14. Disponível em: https://doi.org/10.1590/S0080-6234201400005000018.

10. Santos JLG, Pestana AL, Guerrero P, Meirelles BSH, Erdmann AL. Práticas de enfermeiros na gerência do cuidado em enfermagem e saúde: revisão integrativa. Rev Bras Enferm [Internet]. 2013 [acesso em: 28 nov. 2018];66(2):257-63. Disponível em: https://doi.org/10.1590/S0034-71672013000200016.

11. Soto Fuentes PE, Reynaldos-Grandón K, Martínez-Santana D, Jerez-Yáñez O. Competencias para la enfermera/o en el ámbito de gestión y administración: desafíos actuales de la profesión. Aquichan [Internet]. 2014 [acesso em: 28 nov. 2018];14(1):79-99. Disponível em: https://doi.org/10.5294/aqui.2014.14.1.7.

12. Okagawa FS, Bohomol E, Cunha ICKO. Curso de especialização em gestão em enfermagem: propostas de melhorias segundo discentes. REME Rev Min Enferm [Internet]. 2014 [acesso em: 28 nov. 2018];18(2):320-6. Disponível em: https://doi.org/10.5935/1415-2762.20140025.

13. Almeida E, Piexak DR, Ilha S, Marchiori MRCT, Backers DS. Leadership of the nurse technically responsible: a necessity for the professional practice. Revista de Pesquisa: Cuidado é Fundamental Online [Internet]. 2014 [acesso em: 28 nov. 2018];6(3):998-1006. Disponível em: http://www.seer.unirio.br/index.php/cuidadofundamental/article/view/3069. 14. Peiter CC, Caminha MEP, Oliveira WF. Perfil dos gerentes da Atenção Primária: uma revisão integrativa. Espaço para a saúde - Revista de Saúde Pública do Paraná [Internet]. 2017 [acesso em: 28 nov. 2018];18(1):165-73. Disponível em: http://inesco.org.br/publicacoes/revista/18-1/REpS18-1.pdf.

15. Mendes KDS, Silveira RCCP, Galvão CM. Revisão integrativa: método de pesquisa para a incorporação de evidências na saúde e na enfermagem. Texto Contexto Enferm [Internet]. 2008 [acesso em: 28 nov. 2018];17(4):758-64. Disponível em: https://doi.org/10.1590/S0104-07072008000400018.

16. Melnyk BM, Fineout-Overholt E. Making the case for evidence-based practice and cultivating a spirit of inquiry. In: Melnyk BM, Fineout-Overholt E. Evidence-based practice in nursing \& healthcare: a guide to best practice. A guide to best practice [Internet]. $2^{\text {nd }}$ ed. Philadelphia: Lippincot Williams \& Wilkins; 2005 [acesso em: 28 nov. 2018]. p. 3-24. Disponível em: http://file.zums.ac.ir/ebook/208-Evidence-Based\%20Practice\%20in\%20Nursing\%20\&\%20Healthcare\%20\%20A\%20Guide\%20to\%20Best\%20Practice,\%20Second\%20Edition-Be.pdf.

17. Paula M, Peres AM, Bernardino E, Eduardo EA, Macagi STS. Processo de trabalho e competências gerenciais do enfermeiro da estratégia saúde da família. Rev Rene [Internet]. 2013 [acesso em: 28 nov. 2018];14(4):980-7. Disponível em: http://periodicos.ufc.br/rene/article/view/3633.

18. Melo RC, Machado ME. Coordenação de unidades de saúde da família por enfermeiros: desafios e potencialidades. Rev Gaucha Enferm [Internet]. 2013 [acesso em: 28 nov. 2018];34(4):61-7. Disponível em: https://doi.org/10.1590/S198314472013000400008.

19. Kleba ME, Krauser IM, Vendruscolo C. O planejamento estratégico situacional no ensino da gestão em saúde da família. Texto Contexto Enferm [Internet]. 2011 [acesso em: 28 nov. 2018];20(1):184-93. Disponível em:

https://doi.org/10.1590/S0104-07072011000100022. 
20. Jonas LT, Rodrigues HC, Resck ZMR. A função gerencial do enfermeiro na Estratégia Saúde da Família: limites e possibilidades. Revista de APS [Internet]. 2011 [acesso em: 28 nov. 2018];14(1):28-38. Disponível em:

https://aps.ufjf.emnuvens.com.br/aps/article/view/977.

21. Santos AR. Educação permanente em saúde: construções de enfermeiros da estratégia saúde da família. Revista Baiana de Saúde Pública [Internet]. 2015 [acesso em: 28 nov. 2018];38(3):708-24. Disponível em: https://doi.org/10.22278/23182660.2014.v38.n3.a695.

22. Costa RKS, Miranda FAN. Sistema Único de Saúde e da família na formação acadêmica do enfermeiro. Rev Bras Enferm [Internet]. 2009 [acesso em: 28 nov. 2018];62(2):300-4. Disponível em: https://doi.org/10.1590/S003471672009000200021.

23. Bôas LMFMV, Araújo MBS, Timóteo RPS. A prática gerencial do enfermeiro no PSF na perspectiva da sua ação pedagógica educativa: uma breve reflexão. Cien Saude Colet [Internet]. 2008 [acesso em: 28 nov. 2018];13(4):1355-60. Disponível em: https://doi.org/10.1590/S1413-81232008000400033.

24. Lima FS, Amestosy SC, Jacondino MB, Trindade LM, Silva CN, Fuculo Junior PRB. Exercício da liderança do enfermeiro na estratégia saúde da família. Revista de Pesquisa: Cuidado é Fundamental Online [Internet]. 2016 [acesso em: 28 nov.

2018];8(1):3893-906. Disponível em: https://doi.org/10.9789/2175-5361.2016.v8i1.3893-3906.

25. Paes LG, Bellato TMS, Machado BP, Lima SBS. O uso de indicadores como ferramenta de gestão na estratégia saúde da família. Revista de Enfermagem da UFSM [Internet]. 2015 [acesso em: 28 nov. 2018];5(1):40-9. Disponível em: https://doi.org/10.5902/2179769214150.

26. Costa EMS, Peres AM, Bernardino E, Sade PMC. Estilos de liderança dos enfermeiros que atuam na Estratégia Saúde da Família. Ciênc. cuid. saúde [Internet]. 2015 [acesso em: 28 nov. 2018];14(1):962-9. Disponível em:

https://doi.org/10.4025/cienccuidsaude.v14i1.20756.

27. Rocha BS, Munari DB, Bezerra ALQ, Melo LKA. Enfermeiros coordenadores de equipe do Programa Saúde da Família: perfil profissional. Rev. enferm. UERJ [Internet]. 2009 [acesso em: 28 nov. 2018];17(2):229-33. Disponível em: http://www.facenf.uerj.br/v17n2/v17n2a16.pdf.

28. Sowan AK, Idhail JA. Evaluation of an interactive web-based nursing course with streaming videos for medication administration skills. Int J Med Inform [Internet]. 2014 [acesso em: 28 nov. 2018];83(8):592-600. Disponível em: https://doi.org/10.1016/j.ijmedinf.2014.05.004.

29. Heckemann B, Breimaier HE, Halfens RJ, Schols JM, Hahn S. The participant's perspective: learning from an aggression management training course for nurses. Insights from a qualitative interview study. Scand J Caring Sci [Internet]. 2016 [acesso em: 28 nov. 2018];30(3):574-85. Disponível em: https://doi.org/10.1111/scs.12281.

30. Caveião C, Zagonel IPS, Coelho ICM, Peres AM, Montezeli JH, Venturi KK. Competências na disciplina de administração em enfermagem: pesquisa exploratória descritiva. Online Brazilian J Nurs [Internet]. 2014 [acesso em: 28 nov.

2018];13(4):602-12. Disponível em: https://doi.org/10.5935/1676-4285.20144770.

31. Barbiani R, Nora CRD, Schaefer R. Nursing practices in the primary health care context: a scoping review. Rev Lat Am Enfermagem [Internet]. 2016 [acesso em: 28 nov. 2018];24:e2721. Disponível em: https://doi.org/10.1590/1518-

8345.0880.2721.

32. Ferreira SRS, Périco LAD, Dias VRFG. The complexity of the work of nurses in Primary Health Care. Rev Bras Enferm [Internet]. 2018 [acesso em: 28 nov. 2018];71(supl. 1):704-9. Disponível em: https://doi.org/10.1590/0034-7167-2017-0471. 33. Portaria o 648, de 28 de março de 2006 (BR) [Internet]. Aprova a Política Nacional de Atenção Básica, estabelecendo a revisão de diretrizes e normas para a organização da Atenção Básica para o Programa Saúde da Família (PSF) e o Programa Agentes Comunitários de Saúde (PACS). Diário Oficial da União. 29 mar. 2006 [acesso em: 28 nov. 2018]. Disponível em:

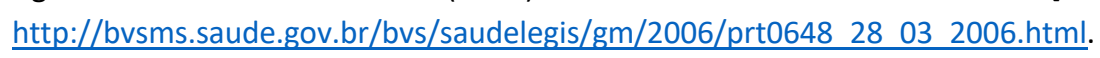

34. Portaria no 2.436, de 21 de setembro de 2017 (BR) [Internet]. Aprova a Política Nacional de Atenção Básica, estabelecendo a revisão de diretrizes para a organização da Atenção Básica, no âmbito do Sistema Único de Saúde (SUS). ). Diário Oficial da União. 22 set. 2017 [acesso em: 28 nov. 2018]. Disponível em:

http://bvsms.saude.gov.br/bvs/saudelegis/gm/2017/prt2436 2209 2017.html.

35. Macêdo NB, Albuquerque PC, Medeiros KR. O desafio da implementação da educação permanente na gestão da educação na saúde. Trab. educ. saúde [Internet]. 2014 [acesso em: 28 nov. 2018];12(2):379-401. Disponível em: https://doi.org/10.1590/S1981-77462014000200010.

36. Salum NC, Prado ML. Continuing education in the development of competences in nurses. Texto Contexto Enferm [Internet]. 2014 [acesso em: 28 nov. 2018];23(2):301-8. Disponível em: https://doi.org/10.1590/0104-

070720140021600011. 
37. Sade PMC, Peres AM. Development of nursing management competencies: guidelines for continuous education services. 0080-6234 [Internet]. 2015 [acesso em: 28 nov. 2018];49(6):988-94. Disponível em: https://doi.org/10.1590/S0080623420150000600016 . 